\title{
BOUNDS IN PIECEWISE LINEAR TOPOLOGY
}

BY

\author{
L. B. TREYBIG( $\left.{ }^{1}\right)$
}

ABSTRACT. The following types of results are obtained: Given a polyhedral 2-sphere $P$ with rectilinear triangulation $T$ lying in the interior of a solid tetrahedron $G$ in $E^{3}$, then there is a simplicial isotopy $f: G \times[0,1] \rightarrow$ $G$ taking $P$ onto a tetrahedron so that for $t$ in $[0,1], f(x, t)=x$ on $\operatorname{Bd}(G)$ and $f_{t}$ is affine on each element of the triangulation $S$ of $G$, where card $(S)$ is a known function of card (T). Also, given (1) $P$ as above, (2) polyhedral disks $D_{1}$ and $D_{2}$, where $\operatorname{Bd}\left(D_{1}\right)=\operatorname{Bd}\left(D_{2}\right) \subset P$ and Int $\left(D_{1}\right) \cup$ Int $\left(D_{2}\right) \subset$ Int $(P)$ and (3) a triangulation $T$ of $D_{1} \cup D_{2} \cup P$, then analogous results are found for a simplicial isotopy $f$ which is fixed on $P$ and takes $D_{1}$ onto $D_{2}$. Given $G$ as above and a piecewise linear homeomorphism $h: G \rightarrow G$ which is fixed on $\mathrm{Bd}(G)$ and affine on each $r \in R$, then analogous bounds are found for a simplicial isotopy $f: G \times[0,1] \rightarrow G$ so that $f_{0}(x)=x$ and $f_{1}(r)=h(r)$ for all $r$ in $R$. In the second half of this paper the normal surface and normal equation theory of Haken is briefly explained and extended slightly. Bounds are found in connection with nontrivial integer entried solutions of normal equations. Also bounds are found for the number of simplexes used in triangulating normal surfaces associated with certain solutions of the extended normal equations.

1. Introduction. The work contained in this paper has grown out of the author's attempts to solve the following problem: Given two oriented polygonal knots $K_{1}, K_{2}$ in regular position in $E^{3}$, show that if $M$ is a solid tetrahedron containing $K_{1}, K_{2}$ in its interior, and $f$ is a piecewise linear homeomorphism taking $M$ onto $M$ and $K_{1}$ onto $K_{2}$ such that (1) $f$ is fixed on $\operatorname{Bd}(M)$, and (2) the orientation of $K_{1}$ induces that of $K_{2}$, then there is a mapping $g$ such that (1) $g$ has the same properties as $f$ (2) $g$ is affine on each simplex of the triangulation $T$ of $M$, and (3) card $T$ is a known function of $n_{1}, n_{2}$ where $n_{i}$ denotes the number of straight line intervals needed to build $K_{i}$. It is evident that such a problem is analogous to a word problem in algebra. This paper is designed to lay some of the needed foundations for attacking the problem. Well-known results on simplicial isotopies [1], [4], [5] and various counting lemmas developed by the author in the present paper and [9] are used to develop

Received by the editors January 19, 1973 and, in revised form, December 20, 1973 and March 15, 1974.

AMS (MOS) subject classifications (1970). Primary 57C15, 55A20; Secondary 55A25, 57 C35.

Key words and phrases. Polyhedral disk or 2-sphere, piecewise linear homeomorphism, simplicial isotopy, normal surface, normal equations.

(1) The author wishes to thank D. J. Hartfiel and D. O'Donnell for helpful suggestions. 
various extensions and improvements of Theorem 1 of Moise [4] and the normal surface and normal equation theory of Haken [3] and Schubert [7].

Let $B(x, y)$ denote a function from a subset of the ordered pairs of nonnegative integers into the positive integers defined by

(0) $B(x, 0)=32^{z-1} 60^{z}$, and

(1) $z=12(2 x-4)(2 x-7) 2^{2 x-2}\left(2 x+(2 x-4)(2 x-7) 2^{2 x-2}\right)$,

(2) $B(x, y)=32^{2} B^{3}(3 x-2, y-1)(32 \cdot 60)^{16(2 x-5)+4 B(3 x-2, y-1)}$

for $4 \leqslant x$ and $1 \leqslant y$. In the present paper the proof of Theorem 1 of [4] is extended to prove

THEOREM 2. Suppose $P$ is a polyhedral 2-sphere which is a subset of the interior of the solid tetrahedron $G$ in $E^{3}$, and that $T$ is a triangulation of $P$ whose elements are rectilinear. Then, there is a simplicial isotopy $f: G \times$ $[0,1] \rightarrow G$ such that (1) $f(x, t)=x$ if $t=0$ or $x \in \operatorname{Bd}(G),(2) f(P, 1)$ is a tetrahedron and (3) there is a rectilinear triangulation $S$ of $G$ such that

(a) $f$ is affine on each $s \in S$ for each $t \in S$ for each $t \in[0,1]$,

(b) $P$ is a subset of the 2-skeleton of $S$, and

(c) if $n$ denotes the number of 0-simplexes of $T$ and $n\left(S_{3}\right)$ the number of 3-simplexes of $S$, then $n\left(S_{3}\right) \leqslant B_{1}(n)$, where $B_{1}(n)=\left(n,\left[2(n-2)^{2} / 3\right]\right)$.

An extension of Theorem 3 of Sanderson [5] which is a consequence of applying Theorem 2 is

THEOREM 3. If $T$ is a triangulation of the polyhedral 3-cell $K$ in $E^{3}$, then there is a subdivision $T^{\prime}$ of $T$ which can be shelled, where if $n=n\left(T_{0}\right)$, the number of 0 -simplexes of $T$, then $n\left(T_{3}^{\prime}\right) \leqslant 96(32) n^{2}\left(T_{3}\right) B_{1}(n)$.

Among the later theorems are (1) Theorem 6, which gives bounds in connection with extending a piecewise linear homeomorphism between two polyhedral 2-spheres to one also between their interiors, and (2) Theorem 7 which gives bounds in connection with realizing a piecewise linear homeomorphism of a solid tetrahedron onto itself which is fixed on the boundary as the final stage of a simplicial isotopy.

In $\S 6$ the normal surface theory of Haken [3] is explained in an abbreviated way. A much more complete description is also found in Schubert [7]. In \$7 the normal equation theory of Haken [3] is explained and extended slightly. In $\S 5$ numerical bounds are found in connection with finding nontrivial integer entried solutions of the extended normal equations. In $\S 8$ bounds are found for the number of simplexes used in triangulating normal surfaces associated with certain solutions of the extended normal equations.

2. Definitions. All spaces considered are subsets of Euclidean 3-space $E^{3}$, 
and all triangulations of such spaces will be locally finite and have closed simplexes which are rectilinear. A subset $S$ of $E^{3}$ will be called a polyhedron, or be said to be polyhedral, if it has a rectilinear triangulation. A mapping $f: S \rightarrow T$ between polyhedra will be said to be piecewise linear (p.l.) if there is a rectilinear triangulation $W$ of $S$ such that $f$ is affine on each $w \in W$. If $f$, $f^{\prime}: S \rightarrow T$ are p.l. homeomorphisms, then $K: S \times[0,1] \rightarrow T$ is a p.l. isotopy between $f$ and $f^{\prime}$ if (0) $K$ is continuous, (1) $K(s, 0)=f(s)$ and $K(s, 1)=$ $f^{\prime}(s)$ for $s \in S$ and (2) for each $t \in[0,1] K_{t}$ is a p.1. homeomorphism. Also, $K$ will be said to be a simplicial isotopy if some fixed triangulation $W$ of $S$ can be found so that $K_{t}$ is affine on each $w \in W$ for each $t \in[0,1]$; and in this case, if $n$ is a positive integer and $S=T$, the ordered pair $(K, W)$ will be said to belong to $M(S, n)$ provided the number of 3-simplexes in $W$ is no more than $n$, and $K(x, t)=x$ if $t=0$ or $x \in \mathrm{Bd}(S)$.

If $T$ is a triangulation of a polyhedron $S$, let $T_{p}(p=0,1,2$ or 3$)$ denote the collection of $p$-simplexes in $T$ and let $n\left(T_{p}\right)=\operatorname{card}\left(T_{p}\right)$. Also for any $T^{\prime} \subset T$ let $\left|T^{\prime}\right|$ denote the union of the elements of $T^{\prime}$. Given $M \subset S$, let $\operatorname{st}(M, T)$ denote the collection of all simplexes of $T$ which contain $M$.

If $M$ is an $n$-manifold with boundary, then $\operatorname{Bd}(M)$ will denote the set of all points of $M$ which do not have a neighborhood in $M$ homeomorphic to $E^{n}$. If $M$ is an $n$-cell, then an $n$-cell $N \subset M$ will be said to be free in $M$ provided $M=N$ or $N \cap \operatorname{Bd}(M)$ is an $n-1$ cell. If $T$ is a cellular subdivision of the $n$-cell $M$ then $M$ can be shelled relative to the elements of $T$ provided they can be labeled $t_{1}, t_{2}, \ldots, t_{m}$ so that if $1 \leqslant p \leqslant m$, then $\bigcup_{i=p}^{m} t_{i}$ is an $n$-cell in which $t_{p}$ is free. (Such an order is called a shelling order.)

If $P \in E^{3}$ and $M \subset E^{3}$, then the cone over $M$ from $P$ (denoted by $P M$ ) is the union of all intervals $P m$, where $m \in M$. Unless otherwise stated, interval means straight line interval, and solid tetrahedron means a tetrahedron plus its interior.

3. Two lemmas and another definition. In the remainder of this paper the following situation occurs frequently.

Lemma 1. In $E^{3}$ let $X$ and $Y$ be solid tetrahedra, where $X \subset \operatorname{Int}(Y)$. Let $f_{1}, f_{2}$ be nonintersecting faces of $X$ such that the sum of their dimensions is two, and let $p_{i}$ be the barycenter of $f_{i}(i=1,2)$. Let $q_{i}(i=1,2)$ be points on the line $p_{1} p_{2}$ in the order $q_{1} p_{1} p_{2} q_{2}$ such that the set $Z$ which is the union of the cones $q_{i} X(i=1,2)$ is a subset of Int $(Y)$. Then there exists $(f, T) \in M(Y, 60)$ such that (1) $f(x, t)=x$ if $t=0$ or $x \in Y-\operatorname{Int}(Z)$, and (2) $f\left(p_{1}, 1\right)=p_{2}$. 
Proof. The transformation is defined as in Theorem 5 of Sanderson [5]. First define $f\left(p_{1}, t\right)=(1-t) p_{1}+t p_{2}$ for $t \in[0,1]$, then define $f$ so that if $z \in \operatorname{Bd}(Z)$ then $f_{t}$ takes interval $z p_{1}$ linearly onto $z f_{t}\left(p_{1}\right)$, and finally define $f_{t}(x)=x$ for $x \in Y-\operatorname{Int}(Z)$.

Let $Q$ denote a triangulation of $\operatorname{Bd}(Z)$ with a minimal number of 2 simplexes. Let $C$ denote the union of all rays $q_{1} z$ where $q_{1} z \cap X=\{z\}$ and let $C_{1}$ and $C_{2}$, respectively, denote the two components of $\operatorname{Int}(Y)-(Z \cup C)$. Use of Lemma 2 of [9] gives triangulations $T^{i}(i=1,2)$ of $\operatorname{Bd}\left(C_{i}\right)$ so that (1) $T^{i}$ agrees with $Q$ on $\operatorname{Bd}(Z)(i=1,2)$, and (2) $n\left(T_{2}^{i}\right) \leqslant 26$. The triangulation $T$ is defined so that if $s$ is a 3-simplex of $T$, then $s$ is of the form $p_{1} w$ for $w \in Q_{2}$, or of the form $s_{i} w$, where $s_{i}$ is a fixed element of $C_{i}$ on line $p_{1} p_{2}$ and $w$ is an element of $T_{2}^{i}$ lying in $\operatorname{Bd}\left(C_{i}\right)$. Since $Z$ contains at most 8 3-simplexes of $T$, the bound above is evident.

Definition. Let a simplicial isotopy $f$ defined as above be called an $S$ isotopy.

Lemma 2. Suppose $X$ is a solid tetrahedron and $\left(f_{i}, T^{i}\right) \in M\left(X, m_{i}\right)$, $i=1, \ldots, n$. Then the transformation $f=f_{n} f_{n-1} \cdots f_{1}$ defined by

$$
f(x, t)=f_{q}\left(f_{q-1}\left(\cdots f_{2}\left(f_{1}(x, 1), 1\right) \cdots 1\right), n t-(q-1)\right) \text { for } x \in X
$$

and $(q-1) / n \leqslant t \leqslant q / n$ has the property that there exists $(f, S) \in$ $M\left(X, 32^{n-1} m_{1} m_{2} \ldots m_{n}\right)$.

PROOF FOR $n=2$. By lemma 4 of [9] there is a subdivision $S$ of $T^{1}$ such that $n\left(S_{3}\right) \leqslant 32 m_{1} m_{2}$ and $\left\{f_{1}(s, 1): s \in S\right\}$ is a refinement of $T^{2}$. Clearly $(f, S)$ is the desired pair.

4. The proofs of the theorems.

THEOREM 1. Suppose $D$ is a polyhedral 2-cell with triangulation $T$ and that $t \in T_{2}$ such that (1) $t \neq D$ and (2) there is $s 0$-simplex $Q$ of $t$ in $\operatorname{Bd}(D)$. Then, if $\epsilon>0$ there is a one-to-one function $f: T_{0} \rightarrow E^{1}$ such that (1) $x \in T_{0}$ implies $\epsilon>f(x) \geqslant 0$, but $f(Q)=0$, (2) if $v \in T_{0} \cap \operatorname{Bd}(D)$ and $v \neq Q$, then there exists $v w \in T_{1}$ such that $f(w)<f(v)$, and (3) if $v \in T_{0} \cap$ Int $(D)$, then there exist elements vw and vu of $T_{1}$ such that $f(u)<f(v)$ $<f(w)$.

Proof. The proof is by induction $n=n\left(T_{2}\right)$.

Case 1: $D=\mid$ st $(Q, T) \mid$ or $n=2$. In this case let the simple closed curve $\operatorname{Bd}(D)$ be denoted by $Q v_{1} v_{2} \ldots v_{n+1} Q$ where $v_{i} \in T_{0}, i=1, \ldots, n+1$. Define $f$ so that $0=f(Q)<f\left(v_{1}\right)<\ldots<f\left(v_{n+1}\right)<\epsilon$. Clearly, $f$ satisfies the conclusion of the theorem.

Case 2: $D \neq \mid$ st $(Q, T) \mid$. Let $n$ be such that if $D^{\prime}$ satisfies the hypothesis 
of the theorem for a triangulation having no more than $n-12$-simplexes, and the simple closed curve $\operatorname{Bd}\left(D^{\prime}\right)$ is labeled $Q v_{1} v_{2} \cdots v_{j} Q$, and a function $g$ is defined such that $0=g(Q)<g\left(v_{1}\right)<\ldots<g\left(v_{j}\right)<\epsilon$, then $g$ may be extended to a function $f$ satisfying the conclusion of the theorem. By Lemma 3 of Sanderson [5] there exist $w \in T_{2}$ such that $Q \notin w$ and $w$ is free in $D$. Let $w=a b c$.

Suppose $w \cap \operatorname{Bd}(D)=a b$. Let the simple closed curve $\operatorname{Bd}(D)$ be labeled $Q v_{1} \ldots a b \ldots v_{j} Q$ and let $g$ map these 0 -simplexes into $E^{1}$ such that $0=$ $g(Q)<g\left(v_{1}\right)<\ldots<g\left(v_{j}\right)<\epsilon$. Extend the domain of $g$ to $c$ so that $g(a)<$ $g(c)<g(b)$. By the induction hypothesis there is an extension of $g$ to a function $f$ satisfying the conclusion of the theorem for $T$ restricted to $\mathrm{Cl}(D-w)$. However, $f$ is the desired function since $f(a)<f(c)<f(b)$.

Suppose $w \cap \operatorname{Bd}(D)=c a \cup b a$. Let $\operatorname{Bd}(D)$ be labeled $Q v_{1} \ldots c a b \ldots$ $v_{j} Q$ and suppose $g$ maps these 0 -simplexes into $E^{1}$ so that $0=g(Q)<$ $g\left(v_{1}\right)<\ldots<g\left(v_{j}\right)<\epsilon$. The restriction of $g$ to $T_{0}-\{a\}$ can be extended to a function $h$ satisfying the conclusion of the theorem for $\mathrm{Cl}(D-w)$, where $h$ is modified slightly so that $h(x) \neq g(a)$ if $x \in T_{0}-\{a\}$. The required function $f$ is defined by $f(x)=h(x)$ if $x \in T_{0}-\{a\}$ and $f(a)=g(a)$.

PROOF OF THEOREM 2. In portions of the following the proof of Theorem 1 of [4] is followed closely. In $E^{3}$ a family $L(u)$ of planes, all normal to a given unit vector $u$, is called admissible if the 0 -simplexes of $T$ lie in different planes of $L(u)$. A typical plane $L$ of $L(u)$ will normally intersect $P$ in the union of a finite collection of disjoint simple closed polygons, but an exceptional plane of $L(u)$ will intersect $P$ in either (1) the union of an isolated point $Q$ and a finite collection of disjoint simple closed polygons, or (2) $n_{P}(L)$ simple closed polygons, $k+1$ of which $\left(1 \leqslant k<n_{p}(L)\right)$ have in common a singular point $Q$, the polygons being otherwise disjoint. Since the isolated and singular points are 0 simplexes of $T$, there are only a finite number of exeptional planes in $L(u)$. Define $n_{P}(u)$ to be $\Sigma n_{P}(L)$, the summation being over planes of $L(u)$ containing singular points. The admissible $L(u)$ 's are determined by the $u$ 's on the unit sphere $S^{2}$ which lie in the complement $C$ of the union of a certain finite collection of great circles. Thus, $C$ is the union of a finite collection of connected open subsets of $S^{2}$, where if $u, u^{\prime}$ belong to the same component of $C$, then $n_{P}(u)=n_{P}\left(u^{\prime}\right)$. Let $s=\min \left\{n_{P}(u), u \in C\right\}$. The proof of the theorem is by double induction on $s$ and $n=n\left(T_{0}\right)$.

In case $s=0$, the minimum for $n$ is 4 , so the triangulation $S$ may be chosen such that $s\left(S_{3}\right) \leqslant 13$. Now suppose the theorem holds for all cases $\left(n^{\prime}, s^{\prime}\right)$ where $1 \leqslant n^{\prime} \leqslant n-1$ and $s^{\prime}=0$, and let $u$ be such that $n_{P}(u)=0$. Let $W$ denote the set of all planes in $E^{3}$ which contain a 2-simplex of $T$, and let $K=\left\{k: k=\bar{w}\right.$, where $w$ is a component of $E^{3}-\bigcup W$ lying in Int $\left.P\right\}$. 
Each $k \in K$ is a convex 3-cell with at most $q=n\left(T_{2}\right)$ flat faces $F$, and each such $F$ is a convex 2-cell bounded by the union of no more than $q-1$ straight line intervals.

Now let $X$ be a triangulation of $P \cup \operatorname{Int} P$ formed as follows: If $F$ is a flat face of a $k$ above, and $F$ is bounded by the union of $j$ straight line intervals (but no fewer), then triangulate $F$ into $j-2$ 2-cells, and then after triangulating all such $F$ s, triangulate each $k$ radially from some interior point of $k$ (see Theorem 2 of [5]) so that the new 3-simplexes are cones over the new 2 simplexes in the $F$ 's above.

Now let $u^{\prime}$ be a close approximation of $u$ lying in the same component of $C$, where no plane of $L\left(u^{\prime}\right)$ contains two 0 -simplexes of $T \cup X$. There are two exceptional planes $L^{\prime}$ and $L^{\prime \prime}$ of $L\left(u^{\prime}\right)$, each containing only one point of $P$, and every plane of $L\left(u^{\prime}\right)$ between $L^{\prime}$ and $L^{\prime \prime}$ intersects $P$ in a simple closed polygon. Let $L^{\prime}=L_{0}, L_{1}, \ldots, L_{j}=L^{\prime \prime}$ be a sequence of planes of $L\left(u^{\prime}\right)$ arranged in their natural order so that (1) for each $i=1, \ldots, j$ exactly one of $L_{i}$ and $L_{i-1}$ contains a 0 -simplex of $T \cup X$, and (2) $T_{0} \cup X_{0} \subset \cup_{i=0}^{i} L_{i}$. Let $Y=\left\{c: c\right.$ is a component of $x-\bigcup_{i=0}^{j} L_{i}$ for some $\left.x \in X_{3}\right\}$. Sanderson's proof of Theorem 2 [5] suffices to show how to find a shelling order of $P \cup \operatorname{Int} P$ relative to the cellular subdivision $Y$. A triangulation $Z$ of $P \cup \operatorname{Int} P$ is now constructed by subdividing each element $z$ of $Z$ into no more than 12 3-simplexes, after first triangulating all flat faces of such $z$ 's with a minimal number of 2-simplexes. The shelling order of $Y$ is then used as in Sanderson [5] to help induce a shelling $m_{1}, m_{2}, \cdots, m_{z}$ of $P \cup \operatorname{Int} P$ relative to $Z_{3}$.

A simple calculation using the Euler characteristic yields $q=n\left(T_{2}\right)=$ $2(n-2)$. The following inequalities may now be established.

(3) $n\left(X_{3}\right) \leqslant q(q-3) 2^{q}$,

(4) $n\left(X_{0}\right) \leqslant q(q-3) 2^{q+2}$,

(5) $j \leqslant 2\left(n\left(T_{0}\right)+n\left(X_{0}\right)\right)$,

(6) $n\left(Y_{3}\right) \leqslant q(q-3) 2^{q}\left(2 n+q(q-3) 2^{q+2}\right)$,

(7) $n\left(Z_{3}\right)=z \leqslant 12 n\left(Y_{3}\right)$.

For each $i(1 \leqslant i \leqslant z-1)$ there exists $\left(f_{i}, S^{i}\right) \in M(G, 60)$ such that (1) $f_{i}$ is an $S$-isotopy, and (2) $f_{i}\left(\bigcup_{p=i}^{z} m_{p}, 1\right)=\bigcup_{p=i+1}^{z} m_{p}$. Lemma 2 is applied to yield $(f, S) \in M(G, B(n, 0))$ where $f=f_{z-1} f_{z-2} \ldots f_{1}$.

Now suppose the bound $B\left(n^{\prime}, t\right)$ has been established for all cases where $0 \leqslant t \leqslant s-1$. Again using Moise's proof as a guide, let $u$ be a unit vector such that $n_{P}(u)=S$. Let $L$ be a singular plane of $L(u)$ and let $Q$ be a singular point of $P$ in $L$. Select a simple closed polygon $J$ of $P \cap L$ which bounds a disk $D$ in $L$, where Int $(D)$ and $P$ are disjoint. $J$ may or may not contain $Q$, but the proof is analogous if it does not. $J$ divides $P$ into two disks $D_{1}$ and $D_{2}$, so let $P_{i}=D \cup D_{i}(i=1,2)$. 
Let the simple closed curve $\operatorname{Bd}(D)$ be denoted by $a_{1} a_{2} \ldots a_{f} a_{1}$, where $a_{1}=Q$ if $Q \in \operatorname{Bd}(D)$, and where the $a_{i}$ 's are the intersections of $D$ with elements of $T_{1}$. If $\operatorname{Bd}(D)$ is a triangle, no further constructions are needed at this stage, so suppose $\operatorname{Bd}(D)$ is not a triangle.

Let $a_{j+1}$ be a point of $\operatorname{Int}(D)$ such that angle $a_{2} a_{1} a_{j+1}$ is acute and $a_{1} a_{2} a_{j+1} \cap \operatorname{Bd}(D)=a_{1} a_{2}$. Since $j \leqslant 2(n-2)$, by Lemma 2 of [9] there is a triangulation $R$ of $D$ so that $(0) a_{1} a_{2} a_{j+1} \in R_{2}$, (1) $\left\{a_{1}, \ldots, a_{j+1}\right\}=$ $R_{0}$, and (2) $n\left(R_{2}\right) \leqslant 2(n-2)$. Let $\epsilon>0$ be such that if $y \in L$ and $v y$ is an interval which is a subset of a 1 -simplex of $T$ or perpendicular to $L$ and the distance from $v$ to $L$ is $\epsilon$, then the length of $v y$ is less than $1 / 10$ the distance between (a) any two planes of $L(u)$ containing 0 -simplexes of $T$, and (b) any two $a_{i}$ 's.

Theorem 1 is now applied to find points $b_{1}, \ldots, b_{j+1}, c_{1}, \ldots, c_{j+1}$ such that (0) $c_{1}=b_{1}=a_{1}$, (1) the $b_{i}$ 's $(i>1)$ lie on one side of $L$ and the $c_{i}$ 's $(i>1)$ on the other, (2) the distances from the $b_{i}$ 's to $L$ satisfy the conclusion of Theorem 1 for the values of $f$, where $Q=a_{1}$; analogously for the $c_{i}$ 's, (3) if $a_{i} \in \operatorname{Int}(D)$ then interval $b_{i} c_{i}$ contains $a_{i}$ and is normal to $L$, and (4) if $a_{i} \in \operatorname{Bd}(D)(i>1)$ then $b_{i} c_{i}$ is a subset of the 1-simplex of $T$ containing $a_{i}$. Given an element $s=a_{p} a_{q} a_{r}$ of $R_{2}$ define the polyhedral 3-cell $A(s)$ to be the set bounded

$$
\begin{gathered}
a_{p} a_{q} a_{r} \cup b_{p} b_{q} b_{r} \cup a_{p} b_{p} a_{q} \cup b_{p} b_{q} a_{q} \cup a_{r} a_{q} b_{r} \cup a_{q} b_{r} b_{q} \cup b_{p} a_{p} a_{r} \\
\cup a_{r} b_{p} b_{r}, \quad \text { where } d\left(a_{q}, b_{q}\right)<d\left(a_{r}, b_{r}\right)<d\left(a_{p}, b_{p}\right),
\end{gathered}
$$

and define $B(s)$ analogously using the $a_{i}$ 's and $c_{i}$ 's.

By Lemma 3 of [5] the elements of $R_{2}-\left\{a_{1} a_{2} a_{j+1}\right\}$ can be shelled from $D$ in some order $r_{1}, r_{2}, \ldots, r_{v}$, and the 3-cells $A\left(r_{1}\right), \ldots, A\left(r_{v}\right)$, $B\left(r_{1}\right), \ldots, B\left(r_{v}\right)$ can be shelled from $P \cup \operatorname{Int} P$ in that order. If each $A\left(r_{i}\right)$ and $B\left(r_{i}\right)$ is triangulated so that the 3-simplexes are formed in each case by starring from some interior point over the triangular disks used to form the boundary, then the shelling order above may be used (see Theorem 2 of [5]) to shell the new tetrahedra from $P \cup \operatorname{Int} P$. Therefore there exist $\left(m_{i}, R^{i}\right) \in$ $M(G, 60), i=1, \ldots, z$, such that (1) each $m_{i}$ is an $S$-isotopy, (2) $z \leqslant 16 V$, and (3) $m_{z} \ldots m_{1}(P, 1)=P^{\prime}$, where $P^{\prime}=\mathrm{Bd}\left(\operatorname{Int} P-\bigcup_{i=1}^{\nu}\left(A\left(r_{i}\right) \cup B\left(r_{i}\right)\right)\right)$. Also, $P^{\prime}$ has a triangulation $T^{\prime}$ such that $T_{0}^{\prime}=\left(P^{\prime} \cap P \cap T_{0}\right) \cup$ $\left\{b_{1}, \ldots, b_{j+1}, c_{1}, \ldots, c_{j+1}\right\}$, and the point $u$ yields an admissible family for $P^{\prime}$ and has an associated integer pair $\left(n^{\prime}, s^{\prime}\right)$, where $n^{\prime} \leqslant 3 n-2$ and $s^{\prime} \leqslant s$.

The disk $a_{1} a_{2} a_{j+1}$ divides $P^{\prime}$ into two open polyhedral 2-cells, $U_{1}$ and $U_{2}$, so let $P_{1}^{\prime}$ denote $a_{1} a_{2} a_{j+1} \cup U_{i}(i=1,2)$. The situation Moise develops at this stage in his proof of Theorem 1 [3] now holds, so it may be seen that 
$P_{i}^{\prime}(i=1,2)$ satisfies the induction hypothesis for the case $\left(n^{\prime}, s^{\prime}\right)$ where $n^{\prime} \leqslant$ $3 n-2$ and $s^{\prime} \leqslant s-1$. The use of Theorem 1 is to avoid the addition of new singular points. Since one of $P_{1}^{\prime}$ and $P_{2}^{\prime}$ is not contained in the 3-cell bounded by the other, suppose $P_{2}^{\prime} \nsubseteq P_{1}^{\prime} \cup \operatorname{Int} P_{1}^{\prime}$.

By the induction hypothesis there exists $\left(f_{i}, S^{i}\right) \in M(G, B(3 n-2, s-1))$ $(i=1,2)$ such that $f_{i}\left(P_{i}^{\prime}, 1\right)$ is a tetrahedron. The number $y$ of 2-simplexes of $S^{1}$ on $P_{1}^{\prime}$ satisfies $y \leqslant 4 n\left(S_{3}^{1}\right)$. By Lemma 3 of [4] there is an ordering $A_{1}, \ldots, A_{y}$ of $\left\{f_{1}(s, 1): s\right.$ is a 2 -simplex of $S_{1}$ on $\left.P_{1}^{\prime}\right\}$ such that (1) $A_{2}, \ldots, A_{y}$ is a shelling of $\mathrm{Cl}\left(f_{1}\left(P_{1}^{\prime}, 1\right)-A_{1}\right)$, and (2) $A_{i}$ which are subsets of $f_{1}\left(a_{1} a_{2} a_{j+i}, 1\right)$ are shelled last $\left(A_{1}\right.$ is not among these). Let $V_{i}=$ Int $f_{1}\left(P_{i}^{\prime}, 1\right)(i=1,2)$, let $x \in V_{1}$, and let $B_{i}$ be the solid tetrahedron $X A_{i}(i=1, \ldots, y)$. There exist $\left(g_{i}, T^{i}\right) \in M(G, 60)(i=1, \ldots, y)$ such that (1) each $g_{i}$ is an $S$-isotopy, and $\bigcup_{q=i+1}^{y} B_{q}$ for $i=1, \ldots, y$.

(2) $g_{i}\left(\bar{V}_{2} \cup \cup_{q=1}^{y} B_{q}, 1\right)=\bar{V}_{2} \cup$

Lemma 2 is now applied to $f=f_{2} f_{1}^{-1} g_{y} \ldots g_{1} f_{1} m_{z} \ldots m_{1}$ to obtain a pair $(f, S) \in M\left(G, 32^{2}(32 \cdot 60)^{y+z} B^{3}(3 n-2, s-1)\right)$ where $y \leqslant$ $4 B(3 n-2, s-1)$ and $z \leqslant 16(2 n-5)$. A simple calculation using the Euler characteristic shows that $n_{P}(u) \leqslant 2 / 3(n-2)^{2}$. Therefore $n\left(S_{3}\right) \leqslant B_{1}(n)$. This completes the proof of Theorem 2.

Proof of THEOREM 3. Let $X$ be a solid tetrahedron such that $K \subset$ $\operatorname{Int}(X)$. By Theorem 2 there is a pair $(f, S) \in M\left(X, B_{1}(n)\right)$ such that $f(K, 1)$ is a solid tetrahedron. By Lemma 4 of [9] there is a common refinement $R$ of $T$, and $S$ restricted to $K$ such that $n\left(R_{3}\right) \leqslant 32 n\left(T_{3}\right) n\left(S_{3}\right)$. Sanderson's proof in Theorem 2 of [4] may be used to find a refinement $W$ of $\{f(r, 1): r \in R\}$ which can be shelled, where $n\left(W_{3}\right) \leqslant 96 n^{2}\left(R_{3}\right)$. The desired triangulation of $K$ is $\left\{f_{1}^{-1}(w): w \in W\right\}$.

TheOREM 4. Suppose $Q$ is a polyhedral 2-sphere in $E^{3}$ and $P_{i}$ $(i=1,2)$ is a polyhedral disk such that

(0) $\operatorname{Bd}\left(P_{1}\right)=\operatorname{Bd}\left(P_{2}\right) \subset Q$,

(1) $P_{1} \cap P_{2}=\operatorname{Bd}\left(P_{1}\right)$

(2) $\operatorname{Int}\left(P_{1}\right) \cup \operatorname{Int}\left(P_{2}\right) \subset \operatorname{Int}(Q)$ (bounded complementary domain), and

(3) $T$ is a triangulation of $P_{1} \cup P_{2} \cup Q$.

Then, there exists $(f, S) \in M\left(Q \cup \operatorname{Int}(Q), 32^{x-1} 60^{x}\right)$ such that

(1) $P_{1} \cup P_{2} \subset\left|S_{2}\right|$

(2) $f\left(P_{1}, 1\right)=P_{2}$, and

(3) $96\left((32(60))^{16 B_{1}(n)} B_{1}(n)\right)^{2}=x$ and $n=n\left(T_{0}\right)$.

Furthermore, if $A X_{i} B(i=1,2)$ is an arc such that $A X_{i} B \subset P_{i} \cap\left|T_{1}\right|$ and $A X_{i} B \cap Q=\{A, B\}$, then there exists $(f, S) \in M\left(Q \cup \operatorname{Int}(Q), 32^{x+y-1} 60^{x+y}\right)$ such that (1) and (2) above hold, (3) $f\left(A X_{1} B, 1\right)=A X_{2} B$, and $f\left(X_{1}, 1\right)=X_{2}$, and 
(4) $N_{1}=28(J+1)(16 J+12), J=2^{5} 5^{3} n^{2}\left(S_{3}\right)(n-1)^{2}$ and $y=$ $(J-1)\left(2 N_{1}+10\right)+9 / 2 J+6 J N_{1}$.

Proof. Let $X$ be a solid tetrahedron such that $Q \subset \operatorname{Int}(X)$. By Theorem 2 there exists $(h, R) \in M\left(X, B_{1}(n)\right)$ such that (1) $h\left(P_{1} \cup P_{2}, 1\right)$ is the boundary of a solid tetrahedron $a b c d$, and (2) $P_{1} \cup P_{2} \subset\left|R_{2}\right|$.

Let $r_{1}, r_{2}, \ldots, r_{w}$ denote an ordering of $\left\{h(r, 1): r \in R_{2}\right.$ and $r \subset P_{1} \cup$ $P_{2}$ \} such that (1) $r_{1} \subset h\left(P_{1}, 1\right)$ but the face of abcd which contains $r_{1}$ does not contain $h\left(P_{2}, 1\right)$, and (2) $r_{2}, \ldots, r_{w}$ is a shelling of $\bigcup_{i=2}^{w} r_{i}$. Suppose, for example, that Int $(a b c)$ intersects $h\left(P_{2}, 1\right)$ but not $r_{1}$. Let $p$ denote the largest integer $t$ such that $\bigcup_{i=t}^{w} r_{i}$ contains $a b c \cup h\left(P_{2}, 1\right)$, let $r_{m_{1}}, \ldots, r_{m_{j}}$ denote a shelling of $\operatorname{Cl}\left(\bigcup_{i=p}^{w} r_{i}-h\left(P_{2}, 1\right)\right)$ from $\bigcup_{i=p}^{r} r_{i}$, and let $r_{n_{1}}, \ldots, r_{n_{k}}$ denote a shelling of $\operatorname{Cl}\left(\bigcup_{i=p}^{w} r_{i}-a b c\right)$ from $\bigcup_{i=p}^{w} r_{i}$. There exist elements $\left(g_{i}, R^{i}\right)$ of $M\left(X, 32(60)^{2}\right), i=1, \ldots, j+k$, such that

(1) $g_{i}(a b c d, 1)=a b c d, 1 \leqslant i \leqslant j+k$,

(2) $\left.g_{s-1}\left(\left(\bigcup_{i=s}^{i} r_{m_{i}}\right) \cup h\left(P_{2}, 1\right), 1\right)=\bigcup_{i=s-1}^{j} r_{m_{i}}\right) \cup h\left(P_{2}, 1\right), 1<s \leqslant$ $j+1$,

(3) $g_{s+j}\left(\left(\cup_{i=s}^{k} r_{n_{i}}\right) \cup a b c, 1\right)=\left(\bigcup_{i=s+1}^{k} r_{n_{i}}\right) \cup a b c, 1 \leqslant s \leqslant k$, and

(4) each $g_{i}$ is an $S$-isotopy or the "composition" of two such, $1 \leqslant i \leqslant$ $j+k$.

By Lemma 2 there exists $(g, V) \in M\left(X,(32 \cdot 60)^{16 B_{1}(n)} B_{1}(n)\right)$ such that $g\left(P_{1} \cup P_{2}, 1\right)=\operatorname{Bd}(a b c d)$ and $g\left(P_{2}, 1\right)=a b c$, where $g=g_{j+k} g_{j+k-1} \ldots$ $g_{j+1} g_{1} g_{2} \ldots g_{j} h$. The techniques of Sanderson in Theorems 2 and 4 of [5] are now applied to find a refinement $U$ of $V^{\prime}=\{g(v, 1): v \in V$ and $g(v, 1) \subset a b c d\}$ whose 3-simplexes can be shelled from $\mathrm{Cl}\left(g\left(\operatorname{Int}\left(P_{1} \cup P_{3}\right)\right), 1\right)$, where $P_{3}$ is the closure of the component $C$ of $Q-\operatorname{Bd}\left(P_{1}\right)$ which is separated from $\operatorname{Int}\left(P_{1}\right)$ by $\operatorname{Int}\left(P_{2}\right)$ in $\mathrm{Cl}(\operatorname{Int}(Q))-\operatorname{Bd}\left(P_{1}\right)$, and where $n\left(U_{3}\right) \leqslant 96 n^{2}\left(V_{3}\right)$. Let $u_{1}, \ldots, u_{x}$ denote such a shelling and let $f_{1}, \ldots, f_{x}$ denote $S$-isotopies such that there exist elements $\left(f_{i}, Z^{i}\right)$ of $M(X, 60), 1 \leqslant i \leqslant x$, such that

$$
\begin{aligned}
f_{i}\left(\bigcup_{s=i}^{x} g^{-1}\left(u_{s}, 1\right) \cup\right. & \left.\mathrm{Cl}\left(\operatorname{Int}\left(P_{2} \cup P_{3}\right)\right), 1\right) \\
& =\bigcup_{s=i+1}^{x} g^{-1}\left(u_{s}, 1\right) \cup \mathrm{Cl}\left(\operatorname{Int}\left(P_{2} \cup P_{3}\right)\right)
\end{aligned}
$$

and $f_{i}(y, t)=y$ if $y \in P_{3}$ and $t \in[0,1], 1 \leqslant i \leqslant x$. By Lemma 2 there is a pair $(f, S) \in M\left(X, 32^{x-1} 60^{x}\right)$, where $f=f_{x} f_{x-1} \ldots f_{1}$. The function $f$ satisfies the conclusions of the first part of the theorem.

By Lemma 4 of [9] there is a triangulation $E^{i}$ of $P_{i}(i=1,2)$ such that (1) $A X_{i} B \subset\left|E_{1}^{i}\right|$, (2) each simplex of $E^{i}$ is a subset of a simplex of $S$, and (3) $n\left(E_{2}^{i}\right) \leqslant 5 \cdot n\left(T_{2}\right) 4 n\left(S_{3}\right)$. Likewise there is a common refinement $E$ of $E^{2}$ 
and $\left\{f(e, 1): e \in E^{1}\right\}$ such that $n\left(E_{2}\right) \leqslant 5 n\left(E_{2}^{1}\right) n\left(E_{2}^{2}\right)$.

The idea is to now move $f\left(A X_{1} B, 1\right)$ onto $A X_{2} B$ by means of a "composition" of $S$-isotopies so as to move $f\left(X_{1}, 1\right)$ onto $X_{2}$. It is straightforward to show the existence of arcs $Y_{1}=f\left(A X_{1} B, 1\right), Y_{2}, \ldots, Y_{J}=A X_{2} B$ such that (1) $J \leqslant 2 n\left(E_{2}\right)$, (2) $Y_{i} \subset\left|E_{1}\right|, Y_{i} \cap \operatorname{Bd}\left(P_{2}\right)=\{A, B\}$, and $Y_{i}$ is an arc from $A$ to $B, 1 \leqslant i \leqslant J$, and (3) there is a sequence $F_{1}, \ldots, F_{J-1}$ of elements of $E_{2}$ such that $Y_{i}-F_{i}=Y_{i+1}-F_{i}$ and $\operatorname{Bd}\left(F_{i}\right) \subset Y_{i} \cup Y_{i+1}, 1 \leqslant$ $i \leqslant J-1$. Consider some fixed $i, 1 \leqslant i \leqslant J-1$, and let $F_{i}=a b c$.

Case 1. Suppose $\dot{Y}_{i} \cap F_{i}=a b$ and $Y_{i+1} \cap F_{i}=a c \cup b c$. There exist elements $a b d, a c d$, and $b c d_{2}$ of $E_{2}$, all distinct from $F_{i}$. Let $e=1 / 2(a+b)$ and let $\epsilon>0$ be such that the solid ball of radius $\epsilon$ centered at $c$ is a subset of $\operatorname{Int}(Q)$ and intersects no simplex of $E-\operatorname{st}(c, E)$. Let $G$ denote the set of all triangular disks $D$ such that there is a 2-simplex $a^{\prime} b^{\prime} c$ in $\operatorname{st}(c, E)$ such that $D=a^{\prime \prime} b^{\prime \prime} c$, where $a^{\prime \prime} c \subset a^{\prime} c$ and is of length $\epsilon$, and $b^{\prime \prime} c \subset b^{\prime} c$ and is of length $\epsilon$. Let $e_{1}, e_{2}$ denote points of line $e c$ in the order $e_{1} e e_{2} c$, where $e_{1} a b d \cap\left(P_{2} \cup Q\right)=a b d$, and $e_{2} \in \operatorname{Int}\left(a_{1} b_{1} c\right)$, where $a_{1} \in a c, b_{1} \in b c$, and $a_{1} b_{1} c \in G$. Let $e_{1}^{\prime}=1 / 3(a+b+d)$.

There exist pairs $\left(w_{i}, W^{i}\right), 1,2, \ldots, 9$, such that

(0) $\left(w_{i}, W^{i}\right) \in M(X, 60)$ and $w_{i}$ is an $S$-isotopy $(1,2,4,5, \ldots, 8)$,

(1) $w_{i}(x, t)=x$ if $x \in \operatorname{Ext}(Q)(i=1,2, \ldots, 9)$,

(2) $w_{1}$ moves $b e_{1}^{\prime} a$ affinely to $b e_{1} a$ and is fixed on $P_{2}-\operatorname{Int}(a b d)$,

(3) $w_{2}$ moves $e$ to $e_{2}$ and moves $a e_{1} e, b e_{1} e, a c e$, and $b c e$ affinely to $a e_{1} e_{2}, b e_{1} e_{2}, a c e_{2}$, and $b c e_{2}$, respectively, and is fixed on $P_{2}-$ $\operatorname{Int}\left(a b e_{1} \cup a b c\right)$,

(4) $w_{3}$ is a "composition" of no more than $N_{1}=28\left(n\left(E_{2}\right)+1\right)$ - $\left(16 n\left(E_{2}\right)+12\right) S$-isotopies such that (a) $w_{3}(|G|, 1)$ is a subset of plane $a b c$ and $w_{3}(x, t)=x$ if $x \in$ disk $a b c$, (b) $w_{3}$ is affine on each $g \in G$ for each $t$ in $[0,1]$ (see Theorem 1 of [9]), and (c) $w_{3}(x, t)=x$ if $x \in P_{2}-|s t(c, E)|$,

(5) $w_{4}$ moves $e_{2}$ to $c$ and moves $a_{2} e_{3} e_{2}$ and $b_{2} e_{3} e_{2}$ affinely onto $a_{2} e_{3} c$ and $b_{2} e_{3} c$, respectively, (where $a_{2}=a_{1} b_{1} \cap a e_{2}, e_{3}=a_{1} b_{1} \cap e c$, and $b_{2}=$ $\left.a_{1} b_{1} \cap b e_{2}\right)$ and $w_{4}(x, t)=x$ for $x \in w_{3}\left(P_{2}-|G|, 1\right)$ and $t \in[0,1]$,

(6) $w_{5}$ and $w_{6}$ play a similar role to $w_{1}, w_{2}$ in that $w_{5}$ moves $1 / 3\left(c+b+d_{2}\right)$ to a point $e_{4}$ on line $b_{2}(1 / 2(b+c))$ close to $1 / 2(b+c)$, and moves no point of $P_{2}-\operatorname{Int}\left(c b d_{2}\right)$, and $w_{6}$ moves $b_{2}$ to $1 / 2(b+c)$ so as to move $c b_{2}$ and $b b_{2}$ affinely onto $c 1 / 2(b+c)$ and $b 1 / 2(b+c)$, respectively, and moves no point of $P_{2}-\operatorname{Int}\left(c e b \cap c b e_{4}\right)$, and $w_{7}$ and $w_{8}$ play a similar role with regard to $c d_{1} a, a_{2}$, and $1 / 2(a+c)$. The transformation

$$
w=w_{7}^{-1} w_{8} w_{7} w_{5}^{-1} w_{6} w_{5} w_{3}^{-1} w_{4} w_{3} w_{1}^{-1} w_{2} w_{1}
$$

is such that (1) $w(x, t)=x$ if $x \in \operatorname{Ext}(Q)$ or $t=0$, (2) $w\left(Y_{i}, 1\right)=Y_{i+1}$, 
$w\left(P_{2}, t\right)=P_{2}$ if $t=0$ or 1 , and (4) $w$ is a "composition" of $10+2 N_{1}$ $S$-isotopies, where the isotopies which compose to form $w_{3}$ also satisfy condition (0) above.

Case 2: $Y_{i} \cap F_{i}=a c \cup b c$. The transformation defined here is simply the inverse of a transformation of the type used in Case 1.

After applying $J-1$ transformations of the types above to obtain a transformation $u$ which moves $Y_{1}$ onto $Y_{J}$, the remainder of the argument involves moving $X_{1}^{\prime}=u\left(f\left(X_{1}, 1\right), 1\right)$ down $Y_{J}$ onto $X_{2}$. Briefly, the idea is to move $X_{1}$ down the appropriate 1 -simplex close to the next 0 -simplex, and then apply a transformation of type $w_{3}$ above which "flattens" a small star neighborhood. Then $X_{1}$ is moved across the 0 -simplex into the next open 1-simplex with the aid of at most two $S$-isotopies, where at the "turn" $X_{1}$ might have to be moved off the track with the first isotopy and back on with the next. The star neighborhood is then returned to its original position with the inverse of the "flattening" map. Since there are no more then $3 J / 2 p$-simplexes $(p=0,1)$ of $E$ on $Y_{2}$, then the number of $S$-isotopies used to move $X_{1}^{\prime}$ to $X_{2}$ with a transformation $v$ need be no more than $3(3 J / 2)+2(3 J / 2)\left(2 N_{1}\right)$. The desired transformation is $v u f$.

THEOREM 4'. If the two bounds stated in Theorem 4 are denoted by $b_{1}(n)$ and $b_{2}(n)$, respectively, and hypothesis $(1)$ is omitted, then $b_{1}(n)$ may be replaced by $32 b_{1}^{2}(w)$ and $b_{2}(n)$ by $32 b_{1}(w) b_{2}\left(20 w b_{1}(w)\right)$, where $w=2 \cdot 6^{2}$. $21 \cdot n(n-1)^{2}$.

Proof. There is a polyhedral 2 cell $P_{3}$ with triangulation $R$ so that (1) $P_{3}$ is "close" to one of the components of $Q-\operatorname{Bd}\left(P_{1}\right)$, (2) $\operatorname{Int}\left(P_{3}\right) \subset \operatorname{Int} Q,(3) P_{3} \cap$ $P_{i}=\operatorname{Bd}\left(P_{1}\right)(i=1,2),(4) R$ and $T$ agree on $\operatorname{Bd}\left(P_{1}\right)$ and (5) the number of 0 simplexes of $R \cup T$ on $\left(P_{1} \cup P_{2} \cup P_{3} \cup Q\right)-\operatorname{Int}\left(P_{i}\right)(i=1,2)$ is $\leqslant w$. Now apply Theorem 4 twice and then Lemma 2 .

THeOREM 5. Suppose $P$ is a polyhedral 2-sphere in $E^{3}, g=a b c d$ is a solid tetrahedron, and $f: \mathrm{Bd}(g) \rightarrow P$ is a p.l. homeomorphism that is affine on each element of the triangulation $T$ of $\operatorname{Bd}(g)$. Then, there is an onto p.l. homeomorphism $h: g \rightarrow P \cup \operatorname{Int}(P)$ and a triangulation $S$ of $g$ such that $(1) f(x)=h(x)$ if $x \in$ $\operatorname{Bd}(g),(2) h$ is affine on each $s \in S$, and $(3) n\left(S_{3}\right) \leqslant 5 \cdot 32 \cdot 4^{2}(n-1)^{2} B_{1}(n)$, where $n=n\left(T_{0}\right)$.

Proof. By Theorem 2 there is an onto p.l. homeomorphism $f_{1}: g \rightarrow P \cup$ $\operatorname{Int}(P)$ and a triangulation $W$ of $g$ so that (1) $f_{1}$ is affine on each $w \in W$ and (2) $n\left(W_{3}\right) \leqslant B_{1}(n)$. Let $W_{2}^{\prime}$ denote the restriction of $W_{2}$ to $\operatorname{Bd}(g)$. Then $n\left(W_{2}^{\prime}\right) \leqslant$ $4 n\left(W_{3}\right)$. By Lemma 4 of [9] there is a refinement $Q$ of $T$ such that $f_{1}^{-1} f: \operatorname{Bd}(g)$ $\rightarrow \operatorname{Bd}(g)$ is affine on each $q \in Q$ and $n\left(Q_{2}\right) \leqslant 5 n\left(T_{2}\right) n\left(W_{2}^{\prime}\right)$. Let $x \in \operatorname{Int}(g)$ and let $Q^{\prime}$ denote the triangulation of $g$ whose 3-simplexes are of the form $x q$, where 
$q \in Q_{2}$. Let $h: g \rightarrow P$ be defined such that if $x q \in Q_{3}^{\prime}$, then $h(x q)=f_{1}\left(x f_{1}^{-1} f(q)\right)$. By Lemma 4 of [9] there is a common refinement $S$ of $W$ and $Q^{\prime}$ such that $n\left(S_{3}\right) \leqslant 32 n\left(W_{3}\right) n\left(Q_{3}^{\prime}\right)$. Clearly, $h$ is affine on each $s \in S$, and if $s \in S$ and $s \subset \operatorname{Bd}(g)$, then $h(s)=f_{1} f_{1}^{-1} f(s)=f(s)$.

THEOREM 6. Suppose $P$ and $Q$ are polyhedral 2-spheres and $f: P \rightarrow Q$ is an onto p.l. homeomorphism which is affine on each simplex of the triangulation $T$ of $P$. Then, there is triangulation $S$ of $P \cup \operatorname{Int}(P)$ and an onto p.l. homeomorphism $g: P \cup \operatorname{Int}(P) \rightarrow Q \cup \operatorname{Int}(Q)$ such that $g$ is affine on each $s \in S, g(x)=f(x)$ if $x \in P$, and

$$
n\left(S_{3}\right) \leqslant 32^{3} \cdot 5^{2} \cdot 4^{4} B_{1}\left((u-1)^{2}\right) B_{1}\left((v-1)^{2}\right)\left(u^{2}-2 u\right)^{2}\left(v^{2}-2 v\right)^{2}
$$

where $u=5\left(4 B_{1}(n)\right)(n-1)^{2}, v=4 B_{1}(n)$, and $n=n\left(T_{0}\right)$.

Proof. By Theorem 2 there is an onto p.l. homeomorphism $g: \operatorname{Bd}(a b c d) \rightarrow Q$ such that (1) $a b c d$ is a solid tetrahedron and (2) $g$ is affine on each simplex $w$ of a triangulation $W$ of $\operatorname{Bd}(g)$, where $n\left(W_{2}\right) \leqslant 4 B_{1}(n)$. Theorem 5 is now applied to $f g^{-1}: \operatorname{Bd}(a b c d) \rightarrow P$ and $g: \operatorname{Bd}(a b c d) \rightarrow Q$ to find extensions $F$ and $G$, respectively. The required map is $G F^{-1}$.

THEOREM 7. If $X$ is a solid tetrahedron and $f: X \rightarrow X$ is an onto p.l. homeomorphism which is affine on each simplex of the triangulation $T$ of $X$, then a bound $b_{3}(n)$ may be stated such that there is an element $(g, S)$ of $M\left(X, b_{3}(n)\right)$ such that (1) $n=n\left(T_{0}\right)$, and $(2) g(r, 1)=f(r)$ for each $r \in T$ if $f(x)=x$ on $\operatorname{Bd}(X)$.

Proof. First apply Theorem 3 to find a subdivision $R$ of $T$ whose 3-simplexes can be given a shelling order $r_{1}, r_{2}, \ldots, r_{i}$. Then Theorem 4 ' is used (the second part of the proof may need to be used twice) to pull $r_{1}$ onto $f\left(f_{1}\right)$ with a simplical isotopy $u_{1}$ so that if $t$ is an $i$-simplex $(i=0,1)$ on $\operatorname{Bd}\left(r_{1}\right)$ then $w_{1}(t, 1)=f(t)$. Likewise Theorem $4^{\prime}$ is used to define $u_{2}$ which pulls $w_{1}\left(r_{2}, 1\right)$ onto $f\left(r_{2}\right)$ in such a way that 0 and 1 simplexes are put in place and $u_{2}$ is fixed on $\operatorname{Bd}(X) \cup$ $f\left(r_{1}\right)$. After $u_{1}, u_{2}, \ldots, u_{i-1}$ have been defined in this way, an application of Lemma 2 completes the proof.

5. Bounds for solutions of certain matrix equations. Let $Z$ denote the integers, let $Z^{\prime}$ denote the nonnegative integers and let $Z^{+}$denote the positive integers. Given a matrix $A=\left(a_{i j}\right)$ with real entries let $\|A\|=\sup \left\{b_{i j} \mid\right\}$.

THEOREM 8. Suppose $A=\left(a_{i j}\right)$ is an $m$ by $n$ matrix, $B=\left(b_{i}\right)$ is an $m$ by 1 matrix, where each entry of $A, B$ is in $Z$ and $\|A\|>0$. Let $b=$ $\sup \{\|A\|,\|B\|\}$ and let $c_{i j} \in Z$ for $1 \leqslant i, j \leqslant n$. Let $N: Z^{+} \times Z^{+} \times Z^{+} \rightarrow Z^{+}$be a function such that

(a) $N(i, 1, k)=k$ and $N(1, j, k) \geqslant N\left(1, j-1, k+k^{2}\right)$ for $i \geqslant 1$ and $j \geqslant 2$, and 
(b) if $i>1$ and $j>1$ then

$N(i, j, k) \geqslant$

$$
\begin{aligned}
\sup \{G=\sup \{ & N(i, j-1, k),\left(1+k N^{i-1}\left(i-1, j-1,2 k^{2}\right)\right) N\left(i-1, j-1,2 k^{2}\right), \\
& (1+j k)\left(j k N\left(i-1, j-1,2 k^{2}\right)\right)+k, \\
& \left.\left.\left.N\left(i, j-1, k+n k^{2} N\left(i-1, j-1,2 k^{2}\right)\right)\right\}, N(i, j-1, k+k G)\right)\right\} .
\end{aligned}
$$

Then, if there is a common solution $X=\left(x_{i}\right)$ of

$$
A X=B \text {, and }
$$

$$
c_{i j} x_{i} x_{j}=0 \quad(1 \leqslant i, j \leqslant n),
$$

where $\|X\|>0$ and each $x_{i} \in Z^{\prime}$, then there is such a solution $Y=\left(y_{i}\right)$, where (a) $0<\|Y\| \leqslant N(m, n, b)$, and (b) $0 \leqslant y_{i} \leqslant x_{i}(1 \leqslant i \leqslant n)$.

PROOF. Since any solution $Y=\left(y_{i}\right)$ of (1) above which satisfies part (b) of the conclusion also satisfies (2), will not be mentioned in the remainder of the proof.

If $n=1$, there is an integer $i$ so that $a_{i 1} \neq 0$. Thus $a_{i 1} x_{1}=b_{i}$ implies that $x_{1}=\left|x_{1}\right| \leqslant\left|b_{i}\right| \leqslant\|B\| \leqslant b$.

Now suppose $m=1, n>1$, and the theorem is valid for all cases $1, \ldots$, $n-1$. Let $Y=\left(y_{i}\right)$ denote a solution of (1) where $\sum_{i=1}^{n} y_{i}$ is a minimum for all solutions $Y$, where $\|Y\|>0$, and each $y_{p} \leqslant x_{p}$. If all $a_{1 i}$ 's are of the same sign, then $\sum_{p=1}^{n}\left|a_{1 p} b_{p}=\right| b_{1} \mid$ so each $y_{p} \leqslant\left|b_{1}\right| \leqslant b$. If two $a_{1 i}$ 's are of opposite sign let $a_{1 i}$ and $a_{1 j}$ be of opposite sign. Either $y_{i} \leqslant\left|a_{1 j}\right|$ or $y_{j} \leqslant\left|a_{1 i}\right|$; for if not, in $Y$ replace $y_{i}$ by $y_{i}-\left|a_{1 j}\right|$ and $y_{j}$ by $y_{j}-\left|a_{1 i}\right|$, and contradict the minimum condition above. Suppose then that $y_{i} \leqslant\left|a_{1 j}\right| \leqslant b$, and consider equation

$$
a_{11} y_{1}+\ldots+a_{1, i-1} y_{i-1}+a_{1, i+1} y_{i+1}+\ldots+a_{1 n} y_{n}=b_{1}-a_{l i} y_{i}
$$

By the induction hypothesis, considering for the moment that in (3) the $y_{p}$ 's $(p \neq i)$ as variable and $y_{i}$ as fixed, there is a solution $Z=\left(z_{p}\right), p \neq i$, such that (a) $0 \leqslant z_{p} \leqslant y_{p}, p \neq i$, and (b) $\sup \left\{z_{p}\right\} \leqslant N\left(1, n-1, b+b^{2}\right)$. Since $y_{i} \leqslant b$, then $\left(z_{1}, \ldots, z_{i-1}, y_{i}, z_{i+1}, \ldots, z_{n}\right)$ is the desired solution.

Before the general inductive step, a definition and lemma are necessary.

Definition. Let $S(A, B)$ denote the set of all solutions $X=\left(x_{i}\right)$ of $A X=B$ so that each $x_{i} \in Z^{\prime}$ and $\|X\|>0$, and let $H(A)$ denote the set all solutions $X=\left(x_{i}\right)$ of $A X=0$ (where 0 is $m$ by 1 if $A$ is $m$ by $n$ ) so that each $x_{i} \in Z^{\prime}$ and $\|X\|>0$. Let $H_{1}(A)$ denote the set of all $X=\left(x_{i}\right)$ in $H(A)$ so that if $X^{\prime}=\left(x_{i}^{\prime}\right)$ is in $H(A)$ and $x_{i}-x_{i}^{\prime} \geqslant 0$ for $: \leqslant i \leqslant n$, then $X=X^{\prime}$. Let $S^{\prime}(A, B)$ denote the set of all $X$ in $S(A, B)$ so that if $Y \in H_{1}(A)$ then $X-Y \notin S(A, B)$. 
Lemma 1. If $X=\left(x_{i}\right)$ is in $S(A, B)(A, B$ as in (3) above), then there exist $C=\left(c_{i}\right)$ in $S^{\prime}(A, B)$, elements $R_{j}=\left(r_{j i}\right)$ of $H_{1}(A) \quad(j=1, \ldots, x)$, and positive integers $a_{1}, \ldots, a_{x}$ such that

$$
X=C+a_{1} R_{1}+\ldots+a_{x} R_{x} .
$$

Proof. The idea of the proof is to keep subtracting elements of $H_{1}(A)$ from $X$ as long as what remains is in $S(A, B)$, and then collect like terms.

Now suppose $m>1, n>1$ and that the theorem has been found valid for all cases $(i, j, b)$ where $i<m$ and $j \leqslant n$ or $i \leqslant m$ and $j<n$.

Now let $Y=\left(y_{i}\right)$ denote a solution of (1) in $S(A, B)$ so that (1) $\|Y\|$ is a minimum $M$, and (2) given $M$, the number of coordinates $y_{i}$ for which $y_{i}=M$ is also a minimum. Assume for example that $y_{1}=\min \left\{y_{1}, \ldots, y_{n}\right\}$.

First note that if the sum of the coefficients in each row' of $A$ is zero then the problem may be solved by rewriting (1) as

$$
a_{11}\left(x_{1}-x_{v}\right)+a_{12}\left(x_{2}-x_{v}\right)+\ldots+a_{1 n}\left(x_{n}-x_{v}\right)=b_{1}
$$

$$
a_{m 1}\left(x_{1}-x_{v}\right)+a_{m 2}\left(x_{2}-x_{v}\right)+\ldots+a_{m n}\left(x_{n}-x_{v}\right)=b_{m}
$$

where $x_{v}=\min \left\{x_{1}, \ldots, x_{n}\right\}$. Noting that $x_{v}-x_{v}=0$, and assuming that each $x_{i}-x_{v}(i \neq v)$ is a variable for the moment, by use of the induction hypothesis there is a solution $W=\left(w_{i}\right)$ of (5) so that each $x_{i}-x_{v} \geqslant w_{i} \geqslant 0$ and

$$
\sup \left\{w_{i}\right\} \leqslant N(m, n-1, b)
$$

Therefore, assume the sum of the coefficients in some row is not zero.

Now assume, for example, that $a_{11} \neq 0$ and derive from $A Y=B$ equations

$$
a_{11} y_{1}=b_{1}-\left(a_{12} y_{2}+\ldots+a_{1 n} y_{n}\right)
$$

$$
\left(a_{11} a_{22}-a_{21} a_{12}\right) y_{2}+\ldots+\left(a_{11} a_{2 n}-a_{21} a_{1 n}\right) y_{n}=a_{11} b_{2}-a_{21} b_{1}
$$

$$
\left(a_{11} a_{m 2}-a_{m 1} a_{12}\right) y_{2}+\ldots+\left(a_{11} a_{m n}-a_{m 1} a_{1 n}\right) y_{n}=a_{11} b_{m}-a_{m 1} b_{1} .
$$

Let (7) be written as a matrix equation

$$
A^{\prime} Y^{\prime}=B^{\prime}
$$

By Lemma 1 and the induction hypothesis 


$$
Y^{\prime}=C+a_{1} R_{1}+\ldots+a_{x} R_{x}
$$

where (1) $C=\left(c_{i}\right), i=2, \ldots, n$, is in $S^{\prime}\left(A^{\prime}, B^{\prime}\right),(2) R_{1}, \ldots, R_{x}$ are distinct elements of $H_{1}\left(A^{\prime}\right)$ such that $R_{p}=\left(r_{p i}\right), i=2, \ldots, n$, (3) sup $\{\|C\|$, $\left.\left\|R_{1}\right\|, \ldots,\left\|R_{x}\right\|, x /(m-1)\right\} \leqslant N\left(m-1, n-1,2 b^{2}\right)$ and (4) each $a_{p}$ is a positive integer.

Now suppose for the moment that $y_{i}=\sup \left\{y_{1}, \ldots, y_{n}\right\}$ and every $R_{p}$ $(1 \leqslant p \leqslant x)$ for which $r_{p i}>0$ is such that $a_{p} \leqslant\left|a_{11}\right|$. Then $y_{i} \leqslant$ $\left(1+x \mid a_{11}\right) N\left(m-1, n-1,2 b^{2}\right)$ or

$$
y_{i} \leqslant\left(1+b N^{m-1}\left(m-1, n-1,2 b^{2}\right)\right) N\left(m-1, n-1,2 b^{2}\right) .
$$

Now suppose that $y_{i}=\sup \left\{y_{1}, \ldots, y_{n}\right\}$ and, for example, that $r_{1 i}>0$ and $a_{1}>\left|a_{11}\right|$. Thus, replace $a_{1}$ by $a_{1}-\left|a_{11}\right|$ to obtain a new element $Y^{\prime \prime}=\left(y_{p}^{\prime \prime}\right), p=2, \ldots, n$, of $S\left(A^{\prime}, B^{\prime}\right)$ and note that $Z^{\prime \prime}=\left(y_{1}^{\prime \prime}, y_{2}^{\prime \prime}, \ldots, y_{n}^{\prime \prime}\right)$ is a solution of (6) where $y_{1}^{\prime \prime}=y_{1}+\left(a_{12} r_{12}+\ldots+a_{1 n} r_{1 n}\right)$, where + is used if $a_{11}>0$, and - is used if $a_{11}<0$.

There are three cases to consider.

Case 1: $0 \leqslant y_{1}^{\prime \prime}<y_{i}$. In this case $Z^{\prime \prime}$ is a solution of $A X=B$ such that (1) $\sup \left\{y_{p}^{\prime \prime}\right\}=y_{i}$ and the number of elements taking on the maximum value is less than the number of $Y$ or (2) $\sup \left\{y_{p}^{\prime \prime}\right\}<y_{i}$. Either case leads to a contradiction.

Case 2: $y_{i} \leqslant y_{1}^{\prime \prime}$. Pick an integer $q$ so that the $q$ th row of $A$ does not sum to zero.

$$
\left|b_{q}-\sum_{p=1}^{n} a_{q p} y_{1}\right| \leqslant \sum_{p=1}^{n}\left|a_{q p}\right| \cdot\left|y_{p}-y_{1}\right|,
$$$$
\left|\sum_{p=1}^{n} a_{q p}\right| y_{1} \leqslant \sum_{p=1}^{n}\left|a_{q p}\right| \cdot\left|y_{p}-y_{1}\right|+\left|b_{q}\right| \text {, }
$$

$$
y_{1} \leqslant \sum_{p=1}^{n}\left|a_{q p}\right| \cdot\left|y_{p}-y_{1}\right|+\left|b_{q}\right|
$$

$$
y_{1}^{\prime \prime} \leqslant\left(1+\sum_{p=1}^{n}\left|a_{q p}\right|\right)\left|a_{12} r_{12}+\ldots+a_{1 n_{1 n}} r_{1 n}\right|+\left|b_{q}\right|
$$

$$
y_{i} \leqslant y_{1}^{\prime \prime} \leqslant(1+n b)\left(n b N\left(m-1, n-1,2 b^{2}\right)\right)+b \text {. }
$$


Case 3: $y_{1}^{\prime \prime}<0$. Thus $y_{1} \leqslant\left|a_{12} r_{12}+\ldots+a_{1 n} r_{1 n}\right|$, or

$$
y_{1} \leqslant n b N\left(m-1, n-1,2 b^{2}\right) \text {. }
$$

Then by considering

$$
a_{12} y_{2}+\ldots+a_{1 n} y_{n}=b_{1}-a_{11} y_{1}
$$

$$
a_{m 2} y_{2}+\ldots+a_{m n} y_{n}=b_{m}-a_{m 1} y_{1}
$$

and assuming for the moment that $y_{2}, \ldots, y_{n}$ are variable, then application of the induction hypothesis to (18) yields a solution $w_{2}, \ldots, w_{n}$ so that $0 \leqslant$ $w_{p} \leqslant y_{p}, p=2, \ldots, n$, and

$$
\sup \left(y_{p}\right)=\sup w_{p} \leqslant N\left(m, n-1, b+b\left(n b N\left(m-1, n-1,2 b^{2}\right)\right)\right) .
$$

It has now been established that there is a solution $Y=\left(y_{i}\right)$ of (1) so that $\sup \left(y_{i}\right)$ is less than the $\sup G$ of the bounds established in (5'), (10), (16), and (19).

Returning to the original problem in the case $(m, n, b)$ let $Y=\left(y_{i}\right) \in$ $S(A, B)$ so that (1) $y_{i} \leqslant x_{i}, i=1, \ldots, n$, and (2) $\|Y\|$ is a minimum for all $Y$ 's satisfying (1). Let $W=\left(w_{i}\right) \in S(A, B)$ be a solution satisfying $\|W\| \leqslant G$. If $w_{i} \leqslant y_{i}$ for all $i$ the bound is established, so suppose $y_{1}<w_{1}$, for example. Then $y_{1}<G$, so consider (18) where $y_{2}, \ldots, y_{n}$ are assumed variable and apply the induction hypothesis to obtain a solution $Y^{\prime}=\left(y_{i}^{\prime}\right), i=2, \ldots, n$, so that $0 \leqslant y_{i}^{\prime} \leqslant y_{i}, i=2, \ldots, n$, and $\sup \left\{y_{p}^{\prime}\right\} \leqslant N(m, n-1, b+b G)$. This completes the proof of Theorem 1 , since $y_{1}, y_{2}^{\prime}, \ldots, y_{n}^{\prime}$ is the desired solution.

6. Normal surfaces. The purpose of this section is to give a brief description of the results of the normalization process described in [3], [7].

Let $M$ be a compact 3-manifold with boundary $\operatorname{Bd}(M)$ and having triangulation $T$. Let $T^{\prime}$ be a cellular decomposition of $M$ where 3-simplexes are polyhedral 3-cells such that (1) the 0 -simplexes of $T$ are contained in a family $B \subset T^{\prime}$ of mutually exclusive "ball" neighborhoods, (2) the parts of the 1 . simplexes of $T$ not contained in $\bigcup_{B}$ are contained in a family $A \subset T^{\prime}$ of mutually exclusive "bar" neighborhoods, (3) the pairs of the 2-simplexes of $T$ not contained in $\bigcup[A \cup B]$ are open 2-cells contained in a family $F \subset T^{\prime}$ of mutually exclusive "flag" neighborhoods, and (4) the closures of components of $M-\bigcup[A \cup B \cup F]$ are a family $R \subset T^{\prime}$ of mutually exclusive "remainder space" neighborhoods. Also assume $\cup A, \cup(A \cup B), \cup(A \cup B \cup F)$ are, respectively, closed neighborhoods of the $0-, 1-$, and 2-skeleton of $T$, and that if two cells 
$x, y$ of $T^{\prime}$ intersect then $x \cap y \in T^{\prime}$, i.e. faces of lower dimension are included in $T^{\prime}$.

If $M \subset E^{3}$ and the elements of $T$ are rectilinear than we may require that the elements of $F \cup R$ be convex, and those of $A, B$ be intersections of convex sets with $M$. Following Waldhausen [10], for each $a \in A$ and each $f \in F$ let $a$ and $f$ be given product structures $D_{a} \times I_{a}$ and $D_{f} \times I_{f}$, respectively, such that (1) the disks of the form $D_{a} \times 0$ and $D_{a} \times 1$ are where $a$ intersects the two adjoining balls, respectively, and (2) if $a$ is determined by $x y$ and $f$ by $x y z$ then an arc of the form $a \cap\left(D_{f} \times t\right)$ is also of the form $p \times[0,1], p \in D_{a}$.

On the boundary of a ball $b$ a set of the form $a \cap b, a \in A$, is called an island; a set of the form $b \cap f, f \in F$, is called a bridge; and a set of the form $b \cap r, r \in R$, is called a lake.

Now let $S$ be a connected 2-manifold with boundary which is piecewise linearly embedded in $M$ so that if $\operatorname{Bd}(S)$ is not void, then $\operatorname{Bd}(S) \subset \operatorname{Bd}(M)$.

Now select some property $\mathbf{P}$ of the surface. The property $\mathbf{P}$ of interest to the author is that " $S$ is an incompressible orientable connected 2-manifold with boundary the fixed simple closed curve $X$ which is a subset of the 1-skeleton of T." Another property suggested by Schubert in [7] is that " $S$ is a 2 sphere in $M$ which does not bound a cell in $M$." We now describe the applicable steps of the normalization process for $S$ as they are given in Schubert [7].

Step 1. By isotopic deformations of $S$, holding $\operatorname{Bd}(S)$ fixed, lift $S-\operatorname{Bd}(S)$ off $\operatorname{Bd}(M)$ so that $S \cap \operatorname{Bd}(M)=\operatorname{Bd}(S)$.

Step 2. Push $S$ out of the elements of $R$. (Unless the word "cut" is used from now on such words as push, pull, etc. will be understood to be movements of $S$ by a homeomorphism of the space.)

Step 3. $\operatorname{Bd}(S)$ is deformed isotopically out of those pieces $f \cap \operatorname{Bd}(M)$, $f \in F$. This isotopy is extended to an isotopy of $S$ so that $S$ still intersects no element of $R$.

Step 4. $S$ is "pulled tight" in the flags by an isotopy which holds $\operatorname{Bd}(S)$ fixed, but so that if $f \in F$ then a component of $S \cap f$ is of the form $D_{f} \times t$.

Step 5. $\operatorname{Bd}(S)$ is isotopically moved on $\operatorname{Bd}(M)$ so that if $x$ is an element of $A$ (respectively, $B$ ) and $y$ is a component of $S \cap \operatorname{Int}(x \cap \operatorname{Bd}(M)$ ), then $y$ is a segment of an arc with endpoints in different elements of $B$ (respectively, $A$ ). The deformation of $\operatorname{Bd}(S)$ is extended to one of $S$. The preceding is done so that if $a \in A, b \in B$, and $x$ is an arc of the form $a \cap b \cap \operatorname{Bd}(M)$, then $\operatorname{Bd}(S)$ crosses $x$ on $\mathrm{Bd}(M)$ at any point they have in common.

Step 6. $S$ is pulled tight in the bars so that if $a \in A$ then a component of $S \cap a$ is of the form $y \times[0,1]$, where $y$ is a simple closed curve in $\operatorname{Int}\left(D_{a}\right)$, or $y$ is an arc lying in $\operatorname{Int}\left(D_{a}\right)$ except for its endpoints, which lie on $\operatorname{Bd}\left(D_{a}\right)$.

Step 7. If there exists $b \in B$ such that some component of $S \cap b$ is not 
a 2-cell, then there is such a component $C$ such that there is a simple closed curve $J$ component of $C \cap \operatorname{Bd}(b)$ and a disk $D$ on $\operatorname{Bd}(b)$ such that (1) $J=$ $\operatorname{Bd}(D)$ and (2) if $J^{\prime}$ is a component of $S \cap \operatorname{Int}(D)$ then $J^{\prime}$ bounds a disk $D^{\prime}$ on $S$, where $\operatorname{Int}\left(D^{\prime}\right) \subset b . S$ is now cut open along a simple closed curve $J_{1}$ on $C$, where $J \cup J_{1}$ bounds an annulus on $C$, and then the two copies of $J_{1}$ are capped by disks whose interiors lie in Int (b), avoid each other, and avoid $S$.

In general, whether or not $S$ is cut into two pieces by this operation, one must check to see if one of the remaining components has property P. If not, the normalization has failed. Note that in the two examples described above normalization does not fail. The process is continued until for all $b \in B$, each component of $b \cap S$ is a 2-cell lying except for its boundary in $\operatorname{Int}(b)$.

Step 8. Suppose $b \in B, r$ is a component of $b \cap \operatorname{Bd}(M)$, and $a \in A$, with $i=a \cap b$ and $a \cap \operatorname{Bd}(M)$ both nonvoid. If there is a component $c$ of $S \cap$ $\operatorname{Bd}(b) \cap \operatorname{Int}(i)$ so that both endpoints of $c$ lie on $r$, then by cut and paste operations such components $c$ will be removed. Such a $c$ is selected so that the endpoints $x$ and $y$ of $c$ on $r$ subtend an arc $x z y$ on $\operatorname{Bd}(i)$ so that $\operatorname{seg}(x z y)$ lies on $\operatorname{Bd}(r)$ and contains no endpoints of any other $c$. Let $g$ be the disk on $i$ bounded by $c \cup x z y$.

$S$ is now cut open on $c$ and capped with disjoint disks $g^{\prime}, g^{\prime \prime}$ one lying close to $g$ in $\operatorname{Int}(b)$ except for an arc on $b \cap \operatorname{Bd}(M)$ and the other lying close to $g$ in Int (a), except for an arc on $a \cap \operatorname{Bd}(M)$. Thus $S \cap \operatorname{Bd}(b)$ has one less component having the properties of $c$.

If the new surface $S^{\prime}$ is still connected we must check to see if $S^{\prime}$ still has property $\mathrm{P}$. If not, the normalization fails. If $S^{\prime}$ has two components $S_{1}$ and $S_{2}$, then one of these has property $P$ or the normalization fails. In the first case $\chi(S)<\chi\left(S^{\prime}\right)$ and in the second case $\chi(S) \leqslant \chi\left(S_{p}\right)$ for $p=1,2$.

Assuming the normalization process does not fail, all components of type $c$ are removed, and the normalization process is repeated from Step 5 on as often as needed until the steps are meaningless for 1 through 8 .

Step 9. Now suppose $b \in B$ and there is a component of $S \cap b$ whose bondary runs more than once across some bridge $y$. Then, there is a component $C$ of $S \cap b$ such that (1) there are two arcs $w_{1}, w_{2}$ of $\operatorname{Bd}(C)$ so that both $w_{1}$ and $w_{2}$ run across $y$ but (2) no point of $S$ lies between them on $y$. There exists $f \in F$ and disks of the form $D_{i}=D_{f} \times t_{i}(i=1,2)$ such that (1) $w_{i} \subset D_{i} \subset S(i=1,2)$ and (2) $f \cap b=y$. There is a disk $D$ on $C$ such that $D \cap \operatorname{Bd}(C)=w_{1} \cup w_{2}$, and a disk $D^{\prime}$ so that (1) $D^{\prime} \subset b$, (2) $\operatorname{Bd}\left(D^{\prime}\right)$ is the union of an arc pqr on $D$ and an arc $p q^{\prime} r$ on $y$ where $p \in \operatorname{seg} w_{1}, r \in$ seg $w_{2}$, segpqr $\subset \operatorname{Int}(D)$, and $p q^{\prime} r \cap w_{1}=p$ and $p q^{\prime} r \cap w_{2}=r$, and (3) $D^{\prime} \cap$ $S=$ pqr. $S$ is now pushed into $f$ along $D^{\prime}$ and then $D_{1}$ and $D_{2}$ are pushed out of $f$ into the adjoining balls and bars. 
Now, starting with Step 6, the process is repeated as long as needed until Step 9 is no longer needed. If property $P$ is retained at each step, then the normalization is completed. A surface for which all the steps are unnecessary is called a normal surface.

Two normal surfaces are regarded as similar if they are isotopic under a map which moves no point out of the cell of $\Sigma^{\prime}$ (dimensions $1,2,3$ ) to which it belongs. Such an isotopy is called inessential.

7. Extended normal equations. Clearly a normal surface $S$ is defined up to inessential isotopy by its intersections with the boundaries of the balls. Such an intersection of $S$ with the boundary of a ball $b$ has components which are simple closed curves $J$ such that if $x$ is an island on $b$ and $y$ is a bridge on $b$ and $z$ and $z^{\prime}$ are disjoint arcs on $J$, then (1) if $z$ is a component of $J \cap x$ then $\operatorname{seg} z \subset \operatorname{Int}(x)$ and the endpoints of $z$ are not on $y$, (2) $J$ is not a subset of $y$, but if $z$ is a component of $J \cap y$ then $z$ runs between the two islands at the ends of $y$, and $z^{\prime} \subset \operatorname{Bd}(b)-y$, and (3) $J$ is not a subset of $x$, but is a subset of the interior of the union of the islands and bridges on $b$. A simple closed curve $J^{\prime}$ on $\operatorname{Bd}(b)$ which is equivalent to $J$ under inessential isotopy is said to belong to the same cut type. The set of all cut type classes is labeled $R_{1}, R_{2}, \cdots, R_{n}$ where $b$ ranges over $B$.

Now suppose $a \in A, b \in B, k \in R_{p}(1 \leqslant p \leqslant n), k \subset b$, and arc $x y$ is a component of $k \cap a \cap b$. The bow type of $x y$ is the set of all $x^{\prime} y^{\prime}$ on $b$ which are equivalent to $x y$ under inessential isotopy. The bow type classes are labeled $k_{1}, \cdots, k_{m}$ where $a$ ranges over $A, b$ over $B$ and $k$ over all $R_{p}$ 's. In [3], [7] the bow type $k_{j}$ is said to be contained in cut type $R_{i}$ if a curve in $R_{i}$ contains an arc in $k_{j}$. Define $a_{j i}=1$ if bow type $k_{j}$ is contained in cut type $R_{i}$ and let $a_{j i}=0$ otherwise.

Let $a \in A$ and let $b_{0}, b_{1}$ be the balls at the ends of $a$. Let the elements of $k_{i}$ lie in $a \cap b_{0}$ and those of $k_{j}$ lie in $a \cap b_{1}$ : If there exists $z \in k_{i}$ such that $y=b_{1} \cap z \times[0,1] \in k_{j}$ then define $b_{i j}=1$ and otherwise let $b_{i j}=0$. If $b_{i j}=1$ then $k_{i}$ and $k_{j}$ are said to be coupled.

Also starting with the notation of the previous paragraph, if for each $z \in$ $k_{i}$ the set $y=b_{1} \cap(z \times[0,1])$ intersects each $x \in k_{j}$ then define $c_{i j}=1$. Otherwise, define $c_{i j}=0$. Professor Haken indicated to the author that he calls $k_{i}$ and $k_{j}$ compatible if $c_{i j}=0$.

We now state some equations satisfied by the surface $S$. Let $x_{i}$ denote the number of times cut type $R_{i}$ occurs on $S$. One condition given in [3], [7] is

$$
b_{j h} \sum_{i=1}^{n}\left(a_{j i}-a_{h i}\right) x_{i}=0 \quad(1 \leqslant j, h \leqslant m),
$$

for each pair of coupled bow types $k_{j}, k_{h}$. Also we have 


$$
c_{j h} a_{j u} a_{h v} x_{u} x_{v}=0 \quad(1 \leqslant j, h \leqslant m ; 1 \leqslant u, v \leqslant n) .
$$

In the case of interest to the author where $\mathrm{Bd}(S)$ is a fixed simple closed curve on $\operatorname{Bd}(M)$, then certain cut types may be specified in advance to occur exactly once. This may be stated as

$$
x_{p_{i}}=1, \quad i=1, \ldots, k .
$$

Now for each $c \in R_{i}$ let $G_{i}^{c}$ denote $c \cap[(\bigcup A) \cap((\bigcup F) \cup \operatorname{Bd}(M))]$ and let $n_{i}=\operatorname{card} G_{i}^{c}$ for some $c$ in $R_{i}$. The surface $S$ is now triangulated (not necessarily rectilinearly) such that (1) all the 0-simplexes lie in $U G_{i}^{c}$, (2) if $k$ is a 2-cell on $S$ bounded by $J \in R_{i}$ then $k$ is subdivided into $n_{i}-2$ 2-simplexes, (3) if $C$ is a component of $S \cap a, a \in A$, then $C$ is subdivided into two 2-simplexes, and (4) if $C$ is a component $S \cap f, f \in F$, then $C$ is subdivided into 4 2-simplexes. The Euler characteristic $\chi$ of $S$ is now computed with the aid of the following equations.

The number of components of the form $S \cap b, b \in B$, is $d=\sum_{i=1}^{n} x_{j}$. The number of components from sets of the form $a \cap S$ is $b=\Sigma_{1}^{n} 1 / 4 x_{j} n_{j}$ since each such component contains four of the 0 -simplexes. The number of components from sets of the form $S \cap f, f \in F$, is $f^{\prime}=(2 b-k) / 3$, where $k$ is defined in (22). Under the triangulation described above the number of 0 -simplexes is

$$
V_{1}=\sum_{1}^{n} x_{j} n_{j}
$$

The number of 1 -simplexes is

$$
S_{1}=\sum_{1}^{n}\left(2 n_{j}-3\right) x_{j}+3 \sum_{1}^{n} 1 / 4 n_{j} x_{j}+3 f^{\prime}
$$

The number of 2-simplexes is

$$
F_{1}=\sum_{1}^{n}\left(n_{j}-2\right) x_{j}+2 b+4 f^{\prime}
$$

and the Euler characteristic $\chi=V_{1}+F_{1}-S_{1}$ is described by

$$
12 \chi=-4 k+\sum_{1}^{n}\left(12-n_{j}\right) x_{j} \text {. }
$$

The extended set of normal equations for $S$ then consists of (20), (21), (22) and (26). Now suppose we consider the set of equations as a defining system for such an $S$. If a nontrivial solution $X=\left(x_{i}\right)$ is found for the system, then it is not hard to verify that a surface $S^{\prime}$ may be built which (1) is in normal position, (2) has the same boundary as $S$, (3) has the same Euler characteristic as $S$, and (4) has the prop- 
erty $x_{i}$ is the number of times cut type $R_{i}$ occurs on $S^{\prime}$. The only problem here is that connectivity may be lost, as can be seen by simple examples. Also remember that the set of equations (20), (21), (22), (26) given here is that of interest to the author. If the reader is interested in some other property $P$, then he would probably have to derive a different set of equations.

8. The number of simplexes used in certain surfaces. In this section the results of $\S \S 5,6$, and 7 are combined to show the existence of triangulated surfaces of a certain "size" provided surfaces of that type exist at all.

Before we proceed we need to make some more definitions. In $E^{3}$ let $M$ be a compact triangulated 3-manifold with boundary and having rectilinear triangulation $T$. We wish to define a specific cellular decomposition $T^{\prime}(M)$.

Let $e_{1}$ denote a positive number less than $1 / 10$ the distance between any two disjoint simplexes of $T$ and let $B_{1}$ denote $\{b$ : there is a 0 -simplex $v$ of $T$ and $\left.b=\left\{x \in M: d(v, x) \leqslant e_{1}\right\}\right\}$. Let $e_{2}$ be a positive number less than $e_{1} / 10$ and such that if $x$ and $y$ belong to disjoint 1-simplexes of $T$ but to no element of $B_{1}$, then $d(x, y)>10 e_{2}$. Let $C_{1}=\left\{x: x \in M\right.$ and $x$ lies within $e_{2}$ of a point of 1skeleton of $T$ \} and let $A_{1}$ denote the set of all closures of components of $C_{1}$ $\left(\cup B_{1}\right)$. Let $e_{3}$ be a positive number less than $e_{2} / 10$ such that if $x, y$ belong to disjoint 2-simplexes of $T$ but not to $\cup\left(A_{1} \cup B_{1}\right)$, then $d(x, y)>10 e_{3}$, and let $C_{2}$ denote $\left\{x: x \in M\right.$ and $x$ lies within $e_{3}$ of the 2-skeleton of $\left.M\right\}$. Let $F_{1}$ denote the set of all closures of components of $C_{2}-\left(\cup\left(A_{1} \cup B_{1}\right)\right)$. Let $R_{1}$ denote the set of all closures of components of $M-\left(\bigcup\left(A_{1} \cup B_{1} \cup F_{1}\right)\right)$.

Given $x \in B_{1}$ let $v_{x}$ denote $\{y: y \in x$ and $y$ belongs to a set of the form $a \cap x \cap \operatorname{Bd}(f)\left(a \in A_{1}\right.$ and $\left.f \in F_{1}\right)$, or $\operatorname{Bd}(a \cup x \cup f) \cap T_{0} \cap \operatorname{Bd}(M)$, or $\left.s \cap P\right\}$ where $s \in T_{1}$ and there exists $a \in A$ such that $P$ is the plane containing $\operatorname{Bd}(a \cap x)-\operatorname{Bd}(M)$, and $s$ determines $a$.

For each $a \in A_{1}$ let $b_{1}, b_{2}$ denote the elements of $B_{1}$ intersecting $a$. Let $v_{a}$ denote $\left\{y: y \in a \cap\left(v_{b_{1}} \cup v_{b_{2}}\right)\right.$ or (if the 1-simplex $s$ which determines $a$ is on $\left.\left.\operatorname{Bd}(M)) y \in s \cap P \cap v_{b_{i}}(i=1,2)\right)\right\}$, where $P$ is as above.

Define $A$ to be $\left\{c(a) \cap M: c(a)\right.$ is the convex hull of $v_{a}$ for some $\left.a \in A_{1}\right\}$. Likewise define $B$. Define $F$ to be the set of all closures of components of $C_{2}-$ $(\cup(A \cup B)$ ), and define $R$ to be the set of all closures of components of $M-$ $(U(A \cup B \cup F))$. Define $R_{1}, \cdots, R_{n}$ as in $\S 7$.

THEOREM 9. In $E^{3}$ let $M$ be a compact 3-manifold with boundary and having rectilinear triangulation $T$. Let $T^{\prime}=T^{\prime}(M)$ be as in the previous definition. Let $S$ be a connected, incompressible, orientable 2-manifold with boundary such that $S \cap \mathrm{Bd}(M)=\mathrm{Bd}(S)$, where $\operatorname{Bd}(S)$ is a simple closed curve which is the union of 1-simplexes of T. Let $S$ have Euler characteristic $\chi$ and let $v=$ the 
number of 0-simplexes of $T$. Then, there is a 2-manifold with boundary $S^{\prime}$ such that (1) $S^{\prime} \cap \operatorname{Bd}(M)=\operatorname{Bd}\left(S^{\prime}\right)=\operatorname{Bd}(S), S^{\prime} \subset M$, and the Euler characteristic of $S^{\prime}$ is $\chi,(2) S^{\prime}$ is in normal position relative to $T^{\prime}$, and (3) there is a vector $Y=\left(y_{i}\right)$ such that (a) $y_{i}$ denotes the number of times cut type $R_{i}$ occurs on $S$, and (b) $\sup \left\{y_{i}\right\} \leqslant N\left(m^{\prime}, n^{\prime}\right.$, sup $\left.\{x, y\}\right)$ where $m^{\prime} \leqslant\left(\begin{array}{c}v \\ 2\end{array}\right) m^{2} n+v+1, n^{\prime} \leqslant v$ !, $x=12+4\left(\begin{array}{c}v \\ 3\end{array}\right), y=12 \chi+4 v+1, m \leqslant 2\left(\begin{array}{c}v \\ 2\end{array}\right)\left[\left(\begin{array}{c}v \\ 3\end{array}\right)\left(\left(\begin{array}{c}v \\ 3\end{array}\right)-1\right)\right], n \leqslant v !$, where $m$ is the number of possible bow types and $n$ the number of possible cut types.

PROof. First note that since $S$ is incompressible the steps for normalization may all be replaced by isotopic deformations so we may as well assume that $S$ satisfies a matrix equation $A^{\prime} X=B^{\prime}$ where the matrices are formed using (20), (22) and (26).

If $n\left(T_{p}\right) \quad(p=0,1,2,3)$ denotes the number of $p$-simplexes of $T$, then $n\left(T_{p}\right) \leqslant\left(\begin{array}{c}v \\ p+1\end{array}\right), p=1,2,3$. At a given 0 -simplex $q$ of $T$, if $b \in B$ and $q \in b$ then the number of cut types on $\operatorname{Bd}(b)$ is at most $(v-1)$ !. Thus, the number $n$ of cut types is no more than $v$ !.

If $a \in A$ and $b \in B$ then the number of bow types on $a \cap b$ is $\leqslant\left(\begin{array}{l}v \\ 3\end{array}\right)\left(\left(\begin{array}{c}v \\ 3\end{array}\right)-1\right)$. Since this bound must be considered at most twice on one 1-simplex, the number $n$ of bow types is $\leqslant 2\left(\begin{array}{c}v \\ 2\end{array}\right)\left[\left(\begin{array}{c}v \\ 3\end{array}\right)\left(\left(\begin{array}{c}v \\ 3\end{array}\right)-1\right)\right]$.

Also the number of $a_{j i}$ is $m n$, and the number of $b_{j h}$ is $\leqslant\left(\begin{array}{c}v \\ 2\end{array}\right) m^{2}$. Also each $n_{j}$ is $\leqslant 4\left(\begin{array}{c}v \\ 3\end{array}\right)$.

Therefore the system of equations (20), (22), (26) as applied to $M, \mathrm{Bd}(S)$, $\chi$ has the property that if it is thought of as a single system as in equation (1), where $A$ is $m^{\prime}$ by $n^{\prime}$ (since $m, n$ are used previously), then $\sup \left\{\left|a_{i j}\right|\right\} \leqslant x=$ $12+4\left(\begin{array}{c}v \\ 3\end{array}\right)$ and $\sup \left\{\left|b_{i}\right|\right\} \leqslant 12 \chi+4 v+1=y$. Also $m^{\prime} \leqslant\left(\begin{array}{c}v \\ 2\end{array}\right) m^{2} n+v+1$ and $n^{\prime} \leqslant v$ !. Therefore there is a solution $Y=\left(y_{i}\right)$ where

$$
\sup \left\{y_{i}\right\} \leqslant N\left(m^{\prime}, n^{\prime}, \sup \{x, y\}\right)
$$

and $y_{i} \leqslant x_{i}$ for each $i$. The surface $S^{\prime}$ is then built using the indicated cut types, then filling in the bars and the flags.

THEOREM 10. In Theorem 9 the surface $S^{\prime}$ may be chosen to have a rectilinear triangulation with at most $(26 / 3) v !\left(\begin{array}{l}v \\ 2\end{array}\right) N\left(m^{\prime}, n^{\prime}, \sup \{x, y\}\right) 2$-simplexes.

Proof. $S^{\prime}$ may be formed so that (1) each disk which is a component of $S^{\prime} \cap b, b \in B$, and determined by cut type $R_{j}$ may be triangulated with $\leqslant n_{j}$ 2-simplexes, where $n_{j}$ is defined in $\S 7,(2)$ each disk which is a component of $A \cap S^{\prime}, a \in A$, may be triangulated with two 2-simplexes, and (3) each component of $S^{\prime} \cap f, f \in F$, may be triangulated with four 2 -simplexes. Therefore the 
total number of 2-simplexes used is less than or equal to

$$
\begin{aligned}
& \sum_{1}^{n} n_{j} x_{j}+2 \sum_{1}^{n} \frac{x_{j} n_{j}}{4}+4 \frac{2 \Sigma_{1}^{n} 1 / 4 x_{j} n_{j}-k}{3} \\
& \leqslant \sum_{1}^{n} \frac{13}{6} n_{j} x_{j} \leqslant \frac{26}{3} v !\left(\begin{array}{l}
v \\
3
\end{array}\right) N\left(m^{\prime}, n^{\prime}, \sup \{x, y\}\right) .
\end{aligned}
$$

This completes the proof of Theorem 10.

\section{REFERENCES}

1. R. H. Bing, An alternative proof that 3-manifolds can be triangulated, Ann. of Math. (2) 69 (1959), 37-65. MR 20 \#7269.

2. R. H. Crowell and R. H. Fox, Introduction to knot theory, Ginn, Boston, Mass., 1963. MR 26 \#4348.

3. W. Haken, Theorie der Normalflächen, Acta Math. 105 (1961), 245-375. MR 25 \#4519a.

4. E. E. Moise, Affine structures in 3-manifolds. II. Positional properties of 2-spheres, Ann. of Math. (2) 55 (1952), 172-176. MR 13, 574.

5. D. E. Sanderson, Isotopy in 3-manifolds. I. Isotopic deformations of 2-cells and 3cells, Proc. Amer. Math. Soc. 8 (1957), 912-922. MR 19, 760.

6. - Isotopy in 3-manifolds. II. Fitting homeomorphisms by isotopy, Duke Math. J. 26 (1959), 387-396. MR 21 \#5956.

7. H. Schubert, Bestimmung der Primfaktorzerlegung von Verkettungen, Math. Z. 76 (1961), 116-148. MR 25 \#4519b.

8. M. K. Fort, Jr. (Editor), Topology of 3-manifolds and related topics, Proc. Univ. of Georgia Inst. (1961), Prentice-Hall, Englewood Cliffs, N.J., 1962. MR 25 \#4498.

9. L. B. Treybig, Concerning a bound problem in knot theory, Trans. Amer. Math. Soc. 158 (1971), 423-436. MR 43 \#4019.

10. F. Waldhausen, Irreducible 3-manifolds which are sufficiently large, Ann. of Math. (2) 87 (1968), 56-88. MR 36 \#146.

DEPARTMENT OF MATHEMATICS, TEXAS A\&M UNIVERSITY, COLLEGE STATION, TEXAS 77843 